\title{
Network topology dynamics of circulating biomarkers and cognitive performance in older Cytomegalovirus-seropositive or -seronegative men and women
}

\author{
Svetlana Di Benedetto ${ }^{1,2}$, Ludmila Müller ${ }^{1 *}$, Stefanie Rauskolb³ ${ }^{3}$ Michael Sendtner ${ }^{3}$, Timo Deutschbein ${ }^{4}$, \\ Graham Pawelec ${ }^{2}$ and Viktor Müller ${ }^{1}$
}

\begin{abstract}
Background: Cytokines are signaling molecules operating within complex cascade patterns and having exceptional modulatory functions. They impact various physiological processes such as neuroendocrine and metabolic interactions, neurotrophins' metabolism, neuroplasticity, and may affect behavior and cognition. In our previous study, we found that sex and Cytomegalovirus (CMV)-serostatus may modulate levels of circulating pro- and anti-inflammatory cytokines, metabolic factors, immune cells, and cognitive performance, as well as associations between them.

Results: In the present study, we used a graph-theoretical approach to investigate the network topology dynamics of 22 circulating biomarkers and 11 measures of cognitive performance in 161 older participants recruited to undergo a sixmonths training intervention. For network construction, we applied coefficient of determination $\left(R^{2}\right)$ that was calculated for all possible pairs of variables $(N=33)$ in four groups ( $C M V$ men and women; $\mathrm{CMV}^{+}$men and women). Network topology has been evaluated by clustering coefficient (CC) and characteristic path length (CPL) as well as local (Elocal) and global ( $E_{\text {global }}$ ) efficiency, showing the degree of network segregation (CC and $E_{\text {local }}$ ) and integration (CPL and $E_{\text {global }}$ ). We found that networks under consideration showed small-world networks properties with more random characteristics. Mean CC, as well as local and global efficiency were highest and CPL shortest in CMV males (having lowest inflammatory status and highest cognitive performance). $\mathrm{CMV}$ and $\mathrm{CMV}^{+}$females did not show any significant differences. Modularity analyses showed that the networks exhibit in all cases highly differentiated modular organization (with Q-value ranged between 0.397 and 0.453).

Conclusions: In this work, we found that segregation and integration properties of the network were notably stronger in the group with balanced inflammatory status. We were also able to confirm our previous findings that CMV-infection and sex modulate multiple circulating biomarkers and cognitive performance and that balanced inflammatory and metabolic status in elderly contributes to better cognitive functioning. Thus, network analyses provide a useful strategy for visualization and quantitative description of multiple interactions between various circulating pro- and anti-inflammatory biomarkers, hormones, neurotrophic and metabolic factors, immune cells, and measures of cognitive performance and can be in general applied for analyzing interactions between different physiological systems.
\end{abstract}

Keywords: Aging, Immunosenescence, Cytomegalovirus, Inflammatory markers, Cytokines, Neurotrophic and metabolic factors, Cognition, Network topology

\footnotetext{
* Correspondence: Imueller@mpib-berlin.mpg.de

${ }^{1}$ Max Planck Institute for Human Development, Berlin, Germany

Full list of author information is available at the end of the article
}

(c) The Author(s). 2019 Open Access This article is distributed under the terms of the Creative Commons Attribution 4.0 International License (http://creativecommons.org/licenses/by/4.0/), which permits unrestricted use, distribution, and reproduction in any medium, provided you give appropriate credit to the original author(s) and the source, provide a link to the Creative Commons license, and indicate if changes were made. The Creative Commons Public Domain Dedication waiver (http://creativecommons.org/publicdomain/zero/1.0/) applies to the data made available in this article, unless otherwise stated. 


\section{Background}

Aging is accompanied by chronic low-grade inflammation that has been repeatedly identified even in overtly healthy individuals and is characterized by elevated levels of circulating pro-inflammatory cytokines [1]. Cytokines represent signaling molecules having exceptional modulatory functions. They impact virtually every physiological process such as neurotransmitter metabolism, neuroendocrine interactions, and neuroplasticity, thereby not only affecting general health but also immunity and cognitive functioning [2-4]. The cytokine network, containing cytokines, their receptors, and their regulators, is present in the brain and in various other physiological systems, and is highly controlled throughout the lifespan [5, 6]. Cytokines and their receptors operate within multifactorial networks and may act synergistically or antagonistically in a time- and concentration-dependent patterns. These interactions allow cross-communication between different cell types, at different hierarchical levels, translating environmental signals into molecular signals $[2,7]$. The proinflammatory profile becomes strategic throughout the lifespan [8-11] - an increase of cytokine secretion, also thought to be associated with the influence of CMVinfection, may be at least partly responsible for ageassociated degenerative disorders [12-16]. Previous studies usually investigated individual roles of different cytokines, inflammatory mediators or metabolic factors in the age-related physiological alterations [17-21]. With growing numbers of biomarkers, however, it may become difficult to interpret results and translate them into useful information.

In our recent work [22], we assessed inflammatory status and cognitive performance in 161 older participants recruited to undergo a six-month training intervention. We demonstrated that sex and CMV-latency have influence on levels of circulating pro- and anti-inflammatory cytokines, receptor antagonist, soluble receptor, metabolic factors, and immune cells. We also found that CMVlatency has modulatory effects on associations between individual peripheral biomarkers [22]. Furthermore, we revealed an interaction between CMV-serostatus and sex associations with cognitive abilities: sex differences in fluid intelligence and working memory were noted only in CMV-negative individuals. Even more strikingly, the same group of elderly men also exhibited a lower inflammatory status in their peripheral circulation. Therefore, a wellbalanced inflammatory and anti-inflammatory equilibrium appeared apparently to be decisive for optimal physiological functions and for optimal cognitive functioning.

Pro-inflammatory cytokines often act as negative regulatory signals modulating the action of hormones and neurotrophic factors. An unbalanced cytokine state may also affect the neuroendocrine system (and vice versa) impairing interplay between them, and contributing to disrupted homeostasis [23]. Therefore, in the present study, we additionally considered such hormones as cortisol and dehydroepiandrosterone (DHEA) as well as neurotrophines and their regulators (insulin-like growth factor-1, IGF-1, and IGF-binding protein, IGFBP-3), to gain a more comprehensive image of these processes. Furthermore, we extended the number of inflammationrelated metabolic factors and included measures of $\mathrm{C}$ reactive protein (CRP) in our present analyses. Finally, instead of focusing on four latent factors representing the main cognitive abilities (as we did in the previous study), we included in our present analysis all 11 individual cognitive performance scores assessed within the cognitive battery of elderly individuals. Increasing complexity arose when attempting to analyze dynamic interconnections between all these factors and to investigate the modulatory impact of CMV-latency and sexual dimorphism. In an effort to better understand the relationships between the multiple circulating and functional biomarkers and to compare them regardless of their physiological hierarchical assignments, we applied a graph-theoretical approach and described constructed networks in terms of network topology and modular organization of network elements.

As stated by Bhavnani et al., network analyses offer two main advantages for studying complex physiological interactions: (i) they do not require a priori assumptions about the relationship of nodes within the data, such as the categorized assumption of hierarchical clustering; and (ii) they allow the simultaneous visualization of several raw values (such as cytokine or/and cell values, functional attributes), as well as aggregated values, and clusters in a uniform visual representation [24]. This allows not only the more rapid generation of hypotheses based on complicated multivariate interactions, but also the validation, visualization, and confirmation of the results, obtained with other methodological approaches. Moreover, this enables a more informed methodology for selecting quantitative methods to compare the patterns obtained in the different sets of data regardless of their physiological hierarchical levels [24].

The purpose of the present study was to visualize and to quantitatively describe by means of a graphtheoretical approach the complex multiple interactions among diverse pro- and anti-inflammatory mediators, immune cell populations, hormones, neurotrophic and metabolic factors as well as cognitive performance in older CMV-seropositive and-negative men and women. Moreover, we aimed to design a new strategy for quantitative investigations of the network topology dynamics in circulating biomarkers and measures of cognitive performance by applying the coefficients of determination $\left(R^{2}\right)$ calculated for all possible pairs of variables in four groups of participants. In order to characterize the 
segregation and integration properties of the individual networks of CMV-positive or -negative men and women, we analyzed such network topology measures as clustering coefficient, characteristic path length, local and global efficiency [25, 26]. With the aim of statistically comparing the network topology dynamics and to identifying the networks with optimal features of segregation and integration, we applied a rewiring procedure. To the best of our knowledge, simultaneous network analyses of multiple inflammation-related peripheral biomarkers and cognitive performance of older Cytomegalovirusseropositive and -seronegative men and women have not been previously accomplished.

\section{Results}

For network analyses, the participants were separated into four groups according to their $\mathrm{CMV}$-serostatus and sex (Fig. 1). For network construction, we applied coefficient of determination $\left(R^{2}\right)$ that was calculated for all possible pairs of variables in four groups $\left(\mathrm{CMV}^{-}\right.$men and women; $\mathrm{CMV}^{+}$men and women). Network topology has been evaluated by clustering coefficient $(C C)$ and characteristic path length $(C P L)$ as well as local $\left(E_{\text {local }}\right)$ and global $\left(E_{\text {global }}\right)$ efficiency (for details see Methods section).

\section{Network composition and network topologies in real and control networks}

Before analyzing network topology changes, we compared the topology in real and control (i.e., lattice and random) networks under different cost levels (the ratio of the number of actual connections to the maximum possible number of connections in the network) in the range between 10 and $60 \%$ of wiring costs. As shown in Additional file 1: Figure 1A, $C C$ is greatest in lattice networks and lowest in random networks, whereas $C C$ for the real networks lies in-between. CPL is shortest in random and longest in lattice networks, while the real networks are between these (see Additional file 1: Figure $1 B)$. Correspondingly, $E_{\text {local }}$ was highest in lattice networks (at least for cost levels under 45\%) and lowest in random networks (at least for cost levels under 20\%), while $E_{\text {global }}$ was highest in random and lowest in lattice networks essentially for all levels of wiring costs, with real networks always in between (see Additional file 1: Figure 2 for details).

Importantly, as shown in Fig. 2, networks under consideration are Small-Word Networks (SWNs) at all levels of wiring costs $(\sigma>1)$. As indicated by the other SW coefficient $\omega$, which is lying at practically all levels of wiring costs in the positive range (see Fig. $2 \mathrm{~b}$ ), these networks are SWNs with more random characteristics. It can also be seen that the networks with costs lower than $25 \%$ showed rather unstable behavior that was stabilizing at the $25 \%$ level of costs and showed very similar results across all experimental groups for both SW coefficients $\sigma$ and $\omega$. Thus, for our main analyses, we decided to set the cost level to $25 \%$ that makes it possible to investigate sparse and at the same time stable network topology in all four groups of participants.

\section{Network structure and network strengths}

It can be seen that connectivity matrices (Fig. 3a) display a group-specific structure in all four participant groups. In the first step, we calculated network strengths as the sum of connections of node $i$ (see also Methods section for more details). As shown in Fig. 3a, b, cognitive nodes exhibit high strengths, which are mostly due to the strong connections between the cognitive nodes themselves, especially in the female groups. In the male groups, the cognitive nodes are also strongly connected to the other systems such as cytokines (especially, in the network of $\mathrm{CMV}^{-}$males), metabolic variables (particularly, in the network of the $\mathrm{CMV}^{+}$males) and immune cells.

\section{Networks of $\mathrm{CMV}^{-}$and $\mathrm{CMV}^{+}$men and women differ in their structure}

Networks of the four experimental groups also display group-specific structure (Fig. 4). Individual nodes (or variables) are represented as multicolored circles coding for affinity to a particular group of variables. The size of the circle depends on the sum of connections and indicates the node's strength. The thickness of the connections corresponds to their connection strength. The nodes are numbered clock-wise beginning from the proinflammatory cytokine IL-1 $\beta$ displayed in blue. The CMV-negative male group (top, left) is characterized by multiple strong connections between pro-inflammatory cytokine nodes (IL-1 $\beta$, TNF, IL-18) and cognitive nodes (episodic memory and fluid intelligence).

Less strong but numerous connections are also present for anti-inflammatory cytokines and the cognitive nodes. Interestingly, this is the only group, in which pro- and anti-inflammatory cytokines show no direct connections to each other. The nodes of perceptual speed are strongly connected with immune cell nodes (lymphocytes and neutrophils). No other groups of participants display such strong direct connections between immune biomarkers and cognition - except the network of $\mathrm{CMV}^{+}$men (bottom, left) with only one strong connection between CRP and fluid intelligence. The network of the $\mathrm{CMV}^{+}$men shows strong connections between metabolic factors and perceptual speed. The network of $\mathrm{CMV}^{-}$women (top, right) displays strong connections between pro-inflammatory IL-6 and triglycerides as well as between anti-inflammatory sTNF-R and creatinine. The network of the $\mathrm{CMV}^{+}$women (bottom right) shows a strong connection between leukocytes and pro- 


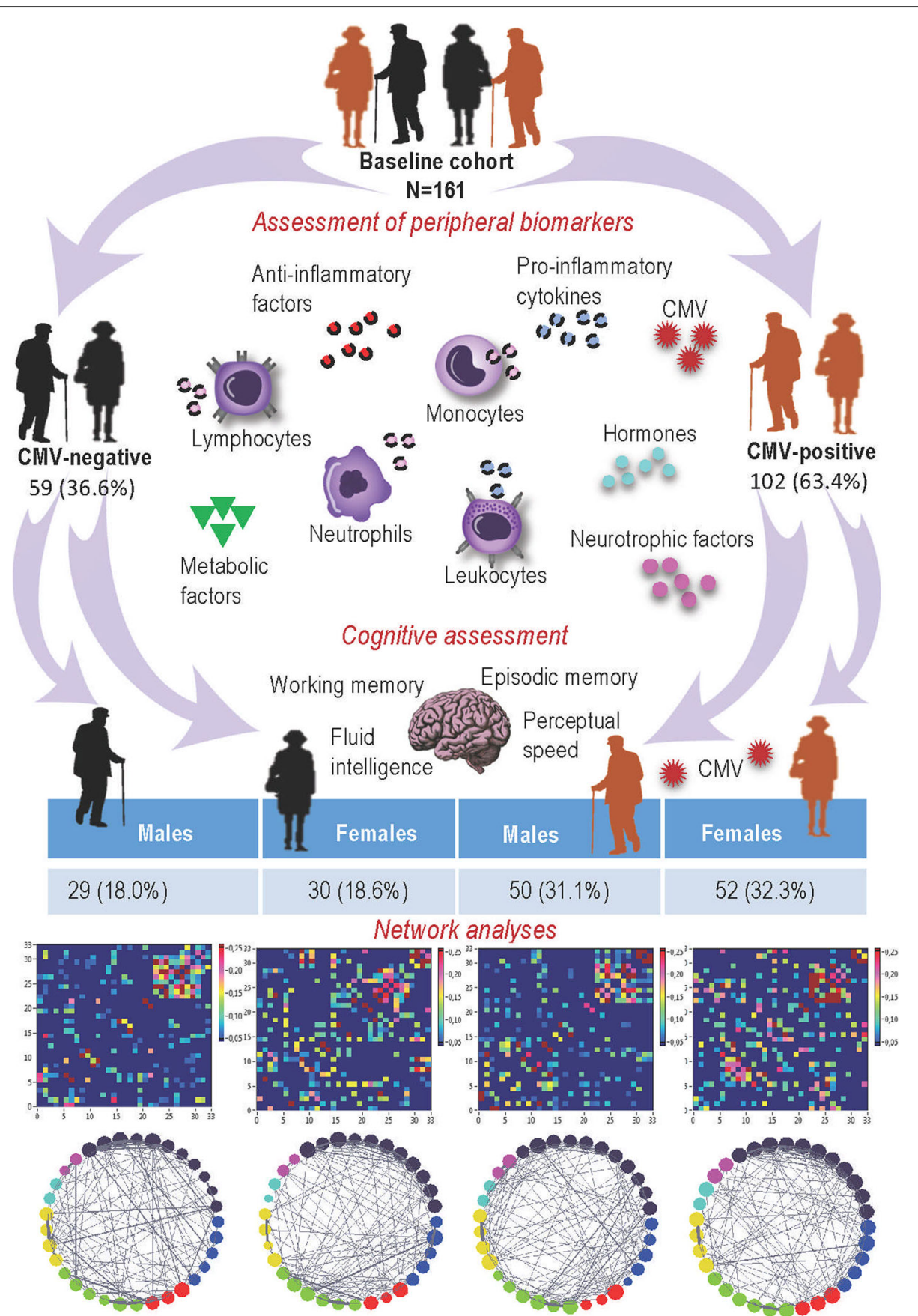

Fig. 1 A schematic illustration of the study setup. Modified from [22]. CMV, Cytomegalovirus

inflammatory IL-6. Unexpectedly, neurotrophins in the $\mathrm{CMV}^{-}$men have relatively strong connections to urea, but only one weak connection to the pro-inflammatory factor CRP. In contrast, all three of the other networks display multiple connections to both pro- and antiinflammatory cytokines. Concerning connections between neurotrophins and cognitive nodes, we can see quite heterogeneous picture: with some connections in CMV-seronegative and -positive men, and with only one connection in the CMV-seronegative and -positive women. In general, the networks of all groups of participants show strong (but differently manifested) connections between the cognitive nodes themselves (Fig. 4).

\section{Networks topology differences between $\mathrm{CMV}^{-}$and $\mathrm{CMV}^{+}$ men and women}

To be able to statistically compare the four different networks at a given cost level, we used rewiring procedure 

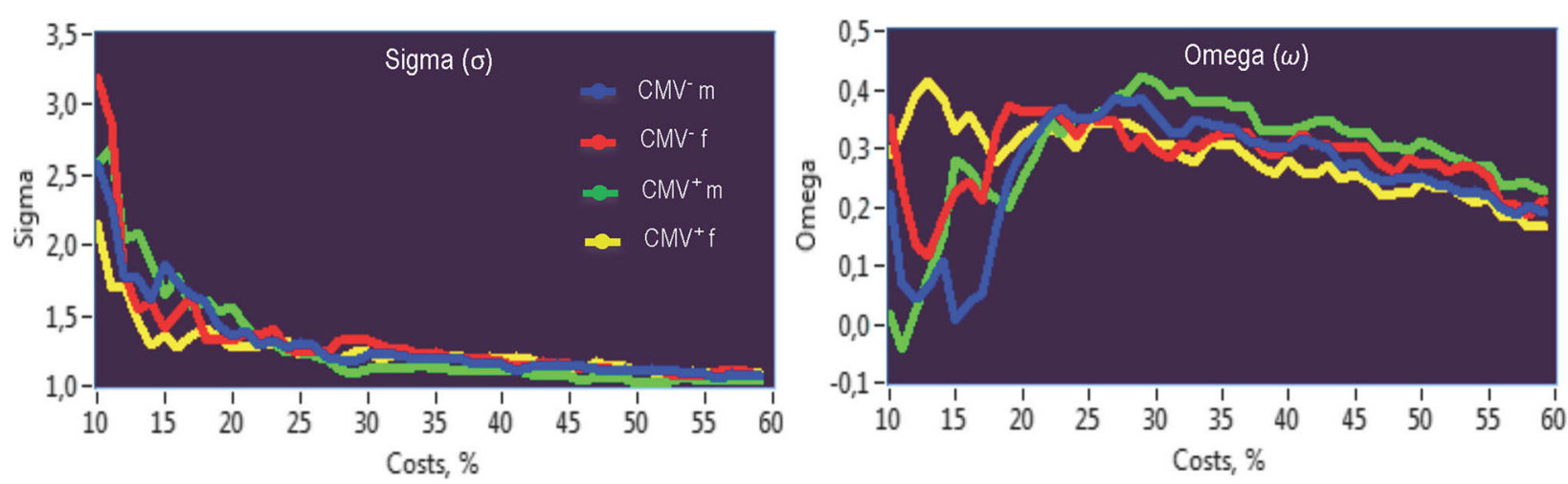

Fig. 2 Small-world coefficients sigma ( $\sigma)$ and omega $(\omega)$ under different levels of the wiring costs. CMV, Cytomegalovirus; CMV ${ }^{-}$, CMVseronegative men; $\mathrm{CMV}^{+} \mathrm{m}$, CMV-seropositive men; $\mathrm{CMV}^{-}$f, $\mathrm{CMV}$-seronegative women; $\mathrm{CMV}^{+}$f, CMV-seropositive women

with replacement of a non-existing edge through an existing one and consecutive determination of network topology metrics each time. In total, there were about 50,000 rewired networks, for which mean and standard deviation (SD) of the network topology metrics were determined. In accordance with the empirical rule, we achieved a $99.7 \%$ confidence interval $(C I)$ for the mean: $C I=$ mean $\pm 3 \times S D$. As shown in Fig. 5 a, mean $C C$ was highest and $C P L$ shortest in $\mathrm{CMV}^{-}$males and in total, higher (shorter) in males than in females. Correspondingly, local and global efficiency were both highest in $\mathrm{CMV}^{-}$males and in total higher in males than in females. CMV-seronegative and -seropositive females did not show any significant differences. This indicates that segregation and integration properties of the network were notably stronger in males (especially, in $\mathrm{CMV}^{-}$ males) than in females. Inspection of separate nodes in the networks showed that these network topology differences were in particular stronger for cytokines and cognitive variables or nodes (Fig. 5b).

\section{Modular organization of the networks of $\mathrm{CMV}^{-}$and $\mathrm{CMV}^{+}$ men and women}

Modularity analyses showed that the networks under consideration exhibited in all cases highly differentiated modular organization with 4 and 5 modules for males and for females, respectively. This is indicated by high modularity values or Q statistics (Fig. 6), which ranged between 0.397 and 0.453 , and were considerably higher as compared with random networks (with $Q$-values close to 0 ). Nodes sharing the same module are displayed in Fig. $6 \mathrm{~b}$ and $\mathrm{d}$ in the same color. As shown in Fig. 6a and c, cognitive nodes occupied two modules in all networks (with exception of $\mathrm{CMV}^{+} \mathrm{fe}-$ males, in which all cognitive nodes were located in one large module), whereby perceptual speed nodes occupied a separate module. Moreover, the community structure in CMV-negative males was organized in 4 modules (A-B, left), whereby all pro-inflammatory cytokines were located in the same module shared (B, blue) with cognitive variables or nodes (reflecting general intelligence and memory features). In addition, two of the three anti-inflammatory cytokines (namely, IL-10 and sTNF-R) shared the same module (B, left, red) with metabolic factors as well as with monocytes, with the exception of urea, which was located in a separate module (B, yellow) together with hormones und neurotrophins. Finally, perceptual speed nodes shared a common module (B, left, green) with IL-1RA and immune cells (namely, leukocytes, lymphocytes, and neutrophils). Interestingly, in $\mathrm{CMV}^{-}$females (A-B, right), the two modules occupied by cognitive ( $\mathrm{B}$, right, blue) and perceptual speed nodes ( $B$, right, cyan) were separated from all the other nodes, which were partitioned into heterogeneous modules comprising different components (e.g., cytokines, metabolic variables, immune cells, and neurotrophins). The nodes of $\mathrm{CMV}^{+}$men $\left(\mathrm{C}-\mathrm{D}\right.$, left) and $\mathrm{CMV}^{+}$women (C-D, right) also partitioned into 4 and 5 modules, respectively, showed heterogeneous modularity structures comprising nodes of both peripheral biomarkers and cognitive features.

\section{$Z-P$ parameter space and nodes' specificity of the four networks}

To define how the network nodes were positioned in their own module and with respect to other modules, we calculated the within-module degree $\left(Z_{i i}\right)$ and participation coefficient $\left(P_{i i}\right)$ of the node $i$ for the given networks. The within-module degree indicates how 'well-connected' node $i$ is to other nodes in the module, whereas the participation coefficient reflects how 'well-distributed' the edges of the node $i$ are among the other modules. $Z_{i}$ and $P_{i}$ form together the so-called $Z-P$ parameter space, with different regions indicating specific roles of the nodes (e.g., hubs, connectors, provincial nodes) in this parameter space [27]. As shown in Fig. 7a, the network of the $\mathrm{CMV}^{-}$ males contains more hub nodes but far fewer connector nodes than the other three groups. This indicates that the modules in this participants' group are more autonomous 


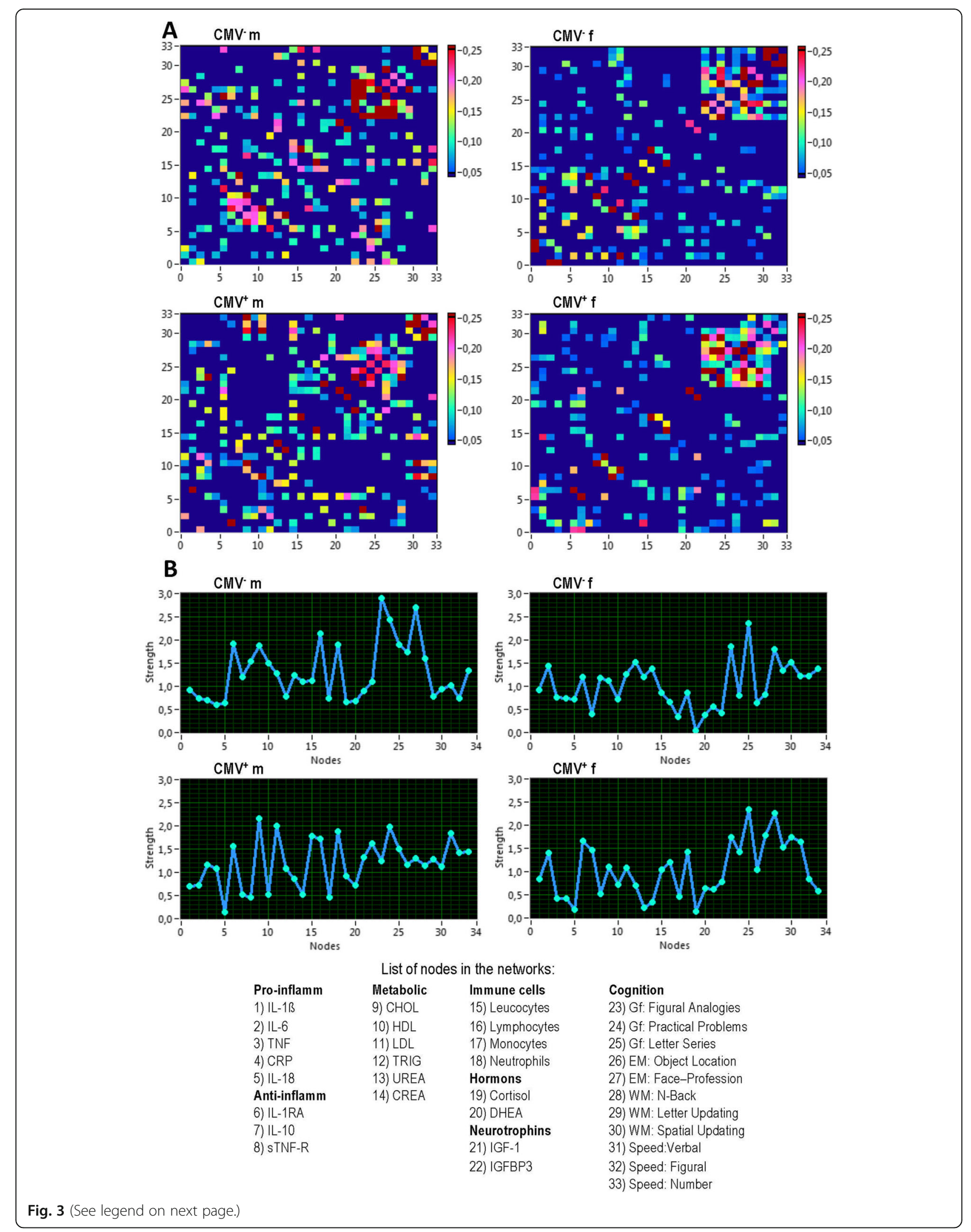


(See figure on previous page.)

Fig. 3 Connectivity structure of the network and network strengths in the four groups. a Connectivity matrices. b Network strengths. CMV, Cytomegalovirus; $\mathrm{CMV}^{-}$m, CMV-seronegative men; $\mathrm{CMV}^{+} \mathrm{m}$, CMV-seropositive men; CMV f, CMV-seronegative women; CMV f, CMV-seropositive women; IL, interleukin; IL-1 $\beta$, interleukin 1 beta; TNF, tumor necrosis factor; CRP, C-reactive protein; IL-1RA, interleukin 1 receptor antagonist; sTNF$\mathrm{R}$, soluble tumor necrosis factor receptor; $\mathrm{CHOL}$, cholesterol; $\mathrm{HDL}$, high-density lipoprotein; LDL, low-density lipoprotein; TRIG, triglyceride; CREA, creatinine; DHEA, dehydroepiandrosterone; IGF-1, insulin-like growth factor-1; IGFBP-3, IGF-binding protein 3; Gf, fluid intelligence; EM, episodic memory; WM, working memory; Speed, perceptual speed

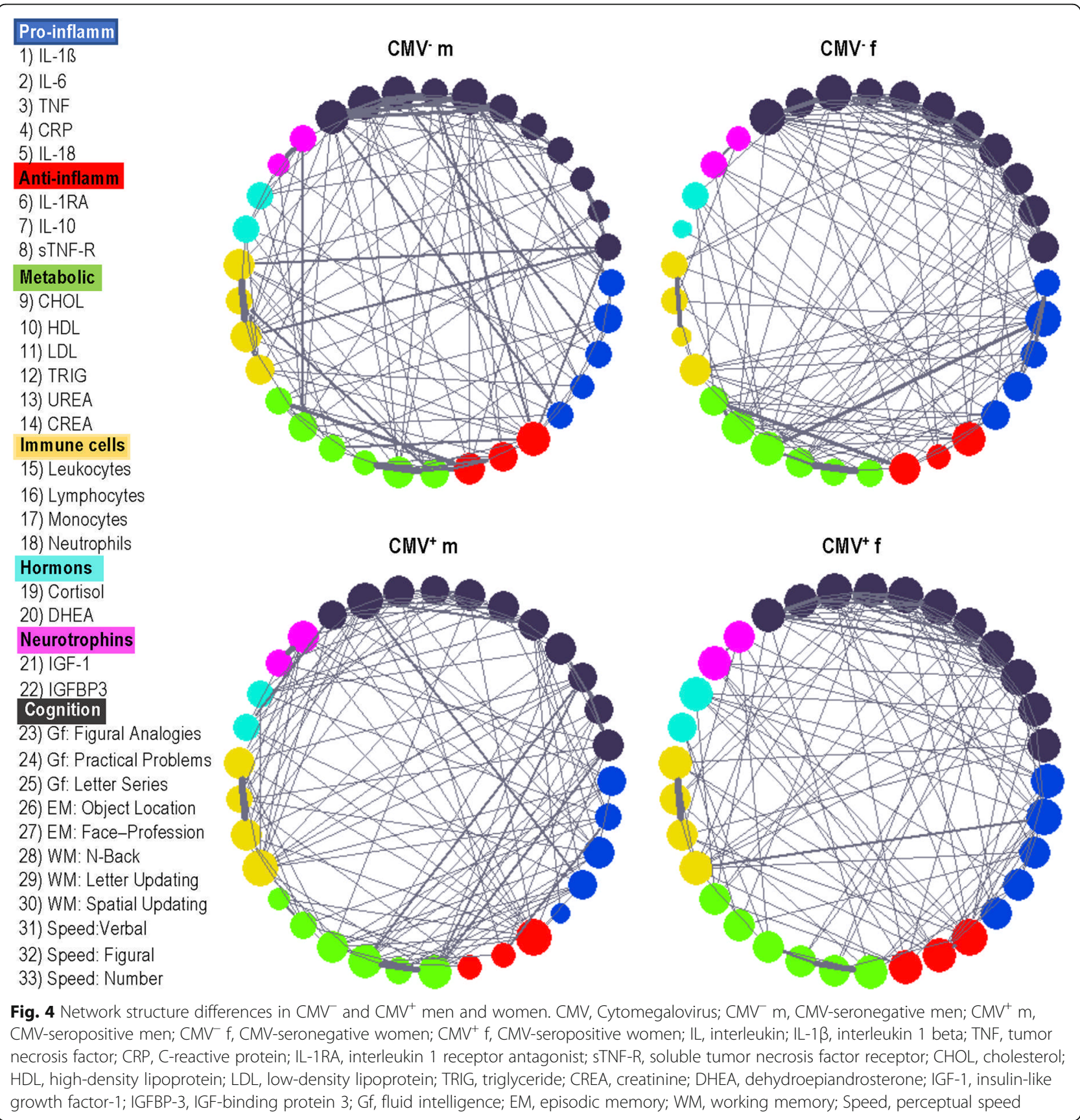




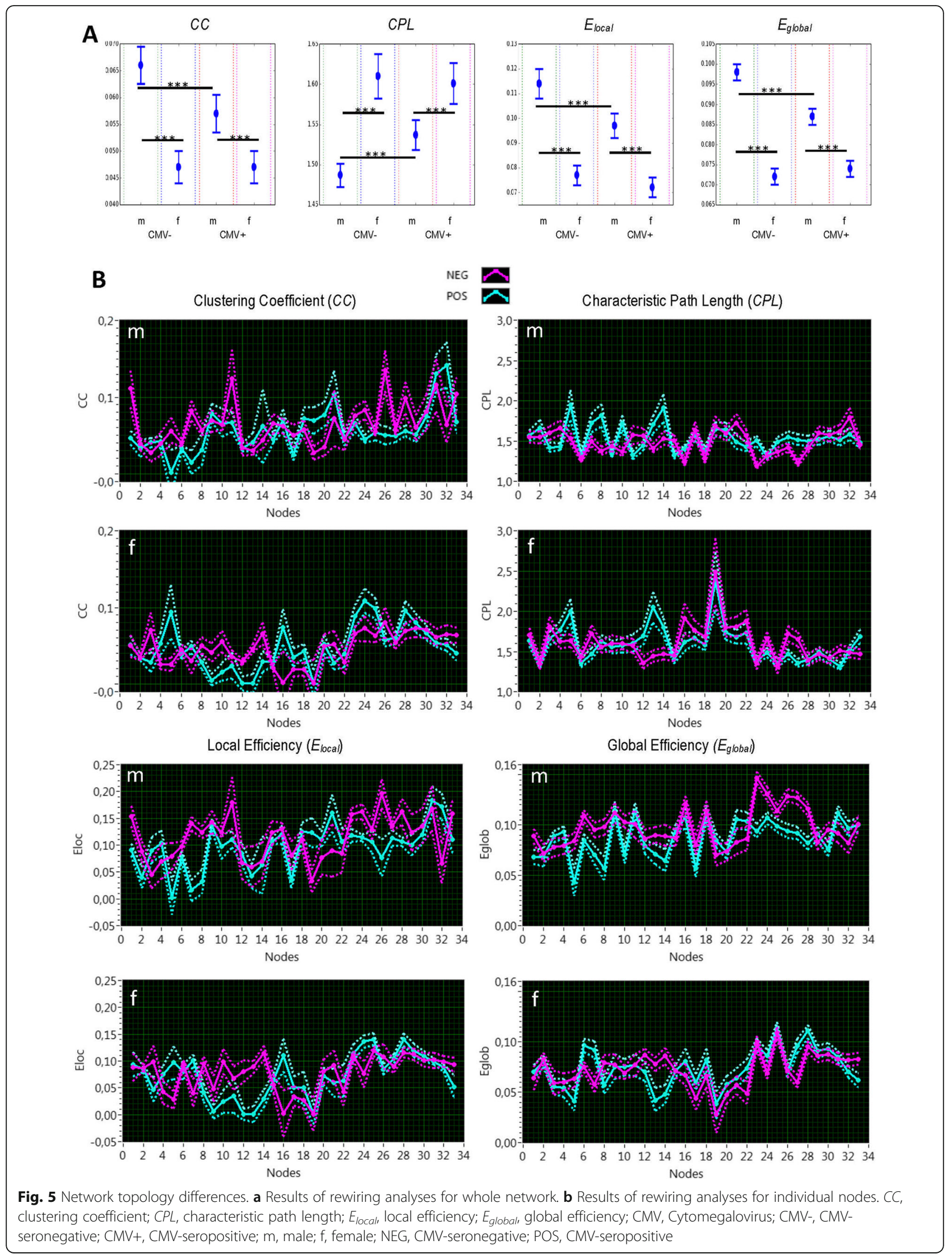



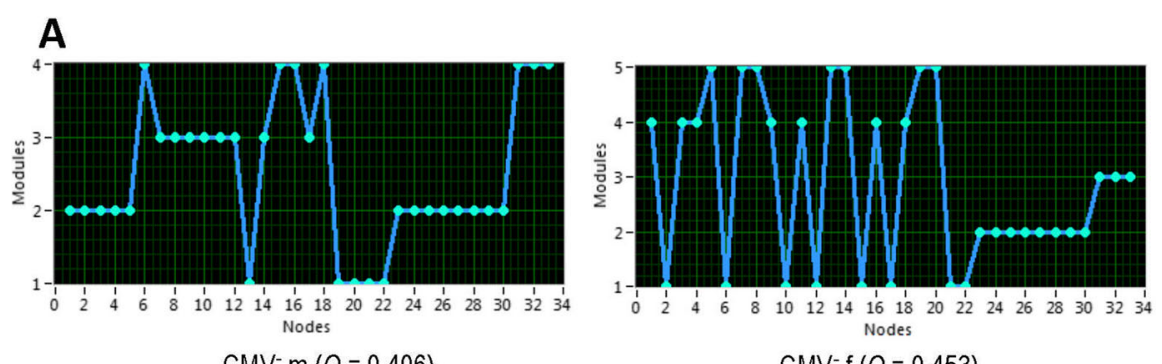

B

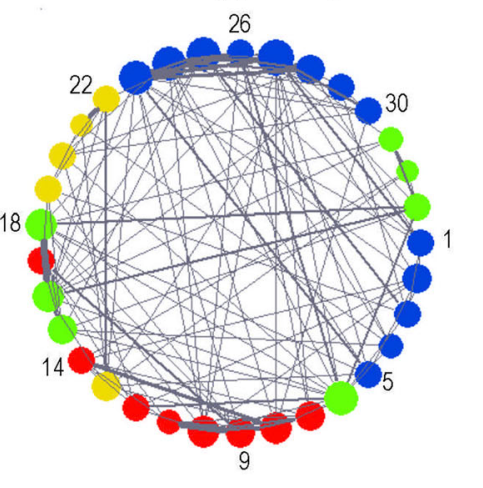

C

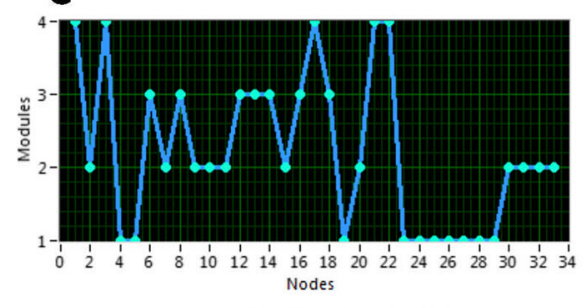

$\mathrm{CMV}^{+} \mathrm{m}(\mathrm{Q}=0.397)$

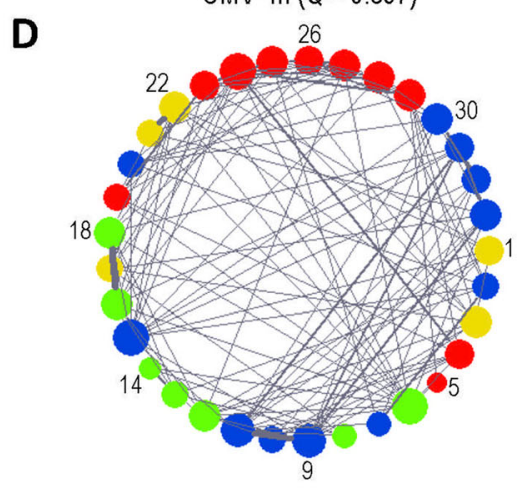

Metabolic

9) $\mathrm{CHOL}$

10) HDL

11) LDL

12) TRIG

13) UREA

14) CREA

Anti-inflamm

6) IL-1RA

7) IL-10

8) STNF-R
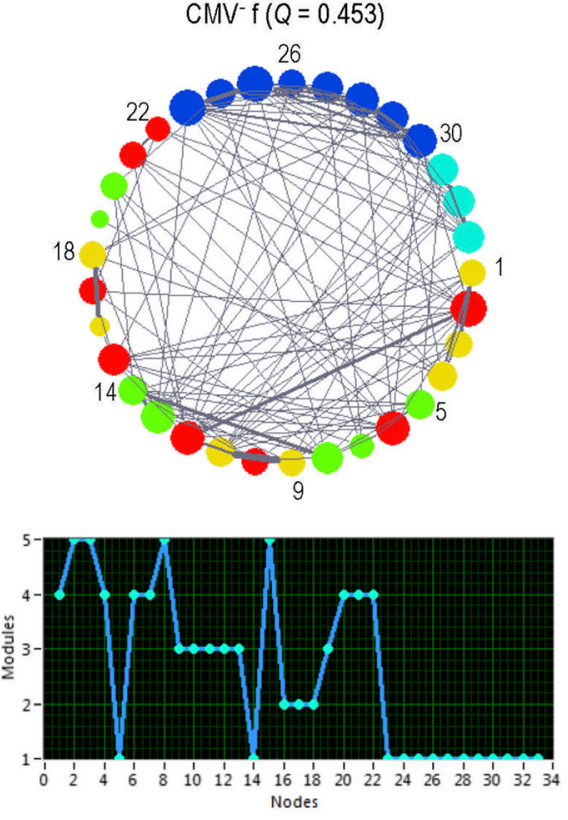

$\mathrm{CMV}^{+} \mathrm{f}(\mathrm{Q}=0.419)$

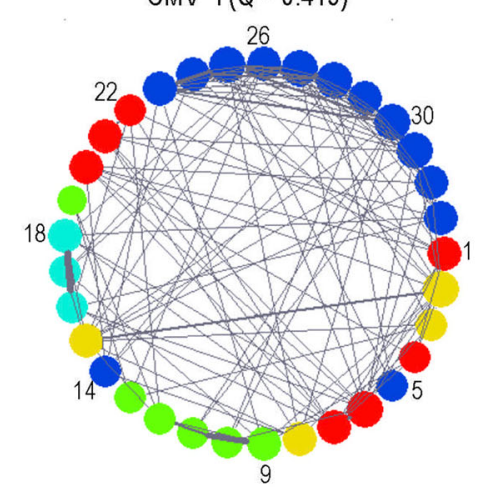

Immune cells

15) Leucocytes

16) Lymphocytes

17) Monocytes

18) Neutrophils

Hormons

19) Cortisol

20) DHEA

Neurotrophins

21) IGF-1

22) IGFBP3
Cognition

23) Gf: Figural Analogies

24) Gf: Practical Problems

25) Gf: Letter Series

26) EM: Object Location

27) EM: Face-Profession

28) WM: N-Back

29) WM: Letter Updating

30) WM: Spatial Updating

31) Speed:Verbal

32) Speed: Figural

33) Speed: Number

Fig. 6 (See legend on next page.) 
(See figure on previous page.)

Fig. 6 Modular organization of the networks. a Modular assignment of nodes in $\mathrm{CMV}^{-}$men (left) and women (right). b Modular structure in $\mathrm{CMV}^{-}$men (left) and women (right). c Modular assignment of nodes in $\mathrm{CMV}^{+}$men (left) and women (right). $\mathbf{d}$ Modular structure in $\mathrm{CMV}^{+}$men (left) and women (right). Note that nodes sharing the same module are displayed in the same color. CMV, Cytomegalovirus; CMV m, CMVseronegative men; $\mathrm{CMV}^{+}$m, CMV-seropositive men; $\mathrm{CMV}^{-}$f, CMV-seronegative women; $\mathrm{CMV}^{+}$f, CMV-seropositive women; $\mathrm{Q}$, modularity value
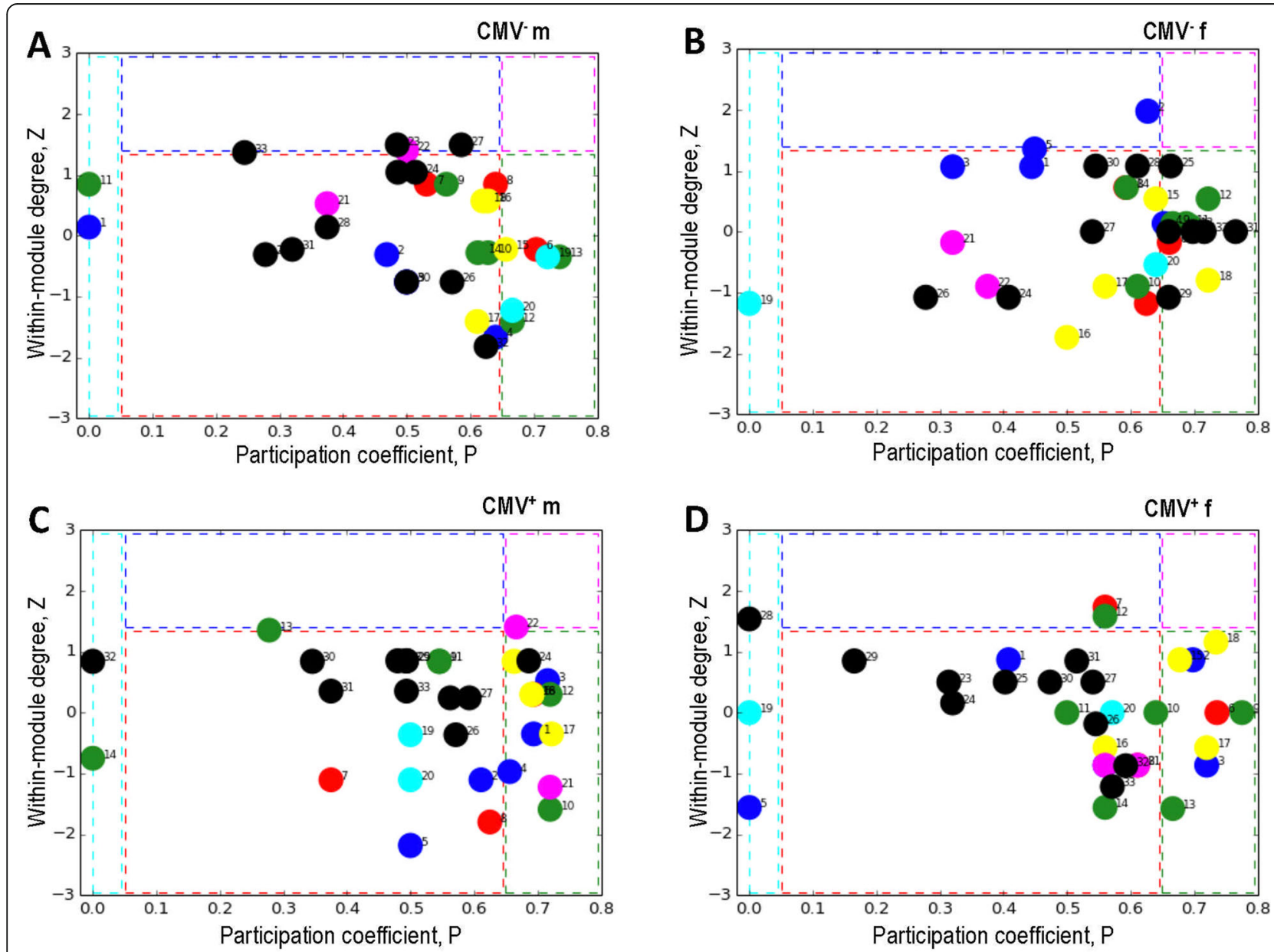

\begin{tabular}{|c|c|}
\hline Pro-inflamm & Metabolic \\
\hline 1) IL-1ß & 9) $\mathrm{CHOL}$ \\
\hline 2) IL-6 & 10) HDL \\
\hline 3) TNF & 11) LDL \\
\hline 4) $\mathrm{CRP}$ & 12) TRIG \\
\hline 5) IL-18 & 13) UREA \\
\hline Anti-inflamm & 14) CREA \\
\hline $\begin{array}{l}\text { 6) IL-1RA } \\
\text { 7) IL-10 } \\
\text { 8) sTNF-R }\end{array}$ & \\
\hline
\end{tabular}

\begin{tabular}{|c|c|}
\hline Immune cells & Cognition \\
\hline 15) Leukocytes & 23) Gf: Figural Analogies \\
\hline 16) Lymphocytes & 24) Gf: Practical Problems \\
\hline 17) Monocytes & 25) Gf: Letter Series \\
\hline 18) Neutrophils & 26) EM: Object Location \\
\hline Hormons & 27) EM: Face-Profession \\
\hline 19) Cortisol & 28) WM: N-Back \\
\hline 20) DHEA & 29) WM: Letter Updating \\
\hline Neurotrophins & 30) WM: Spatial Updating \\
\hline 21) IGF-1 & 31) Speed:Verbal \\
\hline 22) IGFBP3 & $\begin{array}{l}\text { 32) Speed: Figural } \\
\text { 33) Speed: Number }\end{array}$ \\
\hline
\end{tabular}

Fig. 7 Z-P parameter space and node' specificity for networks in four groups. a Z-P parameter space for CMV-seronegative men, (b) Z-P parameter space for CMV-seronegative women, (c) Z-P parameter space for CMV-seropositive men, and (d) Z-P parameter space for CMVseropositive women. Different regions separated by dotted lines contain: left - ultra-peripheral nodes; central - provincial nodes; top - hubs; top right - connector hubs; right - connectors. CMV, Cytomegalovirus; CMV m, CMV-seronegative men; $\mathrm{CMV}^{+} \mathrm{m}$, CMV-seropositive men; CMV f CMV-seronegative women; $\mathrm{CMV}^{+}$f, CMV-seropositive women 
and the information flow between the modules is either reduced or is realized through a small number of connector nodes. Interestingly, three of the four hubs are cognitive variables and the fourth one is IGFBP3. Thus, cognitive nodes, such as fluid intelligence, working memory, and perceptual speed, play a central role in the network of $\mathrm{CMV}^{-}$males driving or controlling the connections within the corresponding modules. Further, the networks of $\mathrm{CMV}^{-}$females (B) and $\mathrm{CMV}^{+}$males (C) are characterized by high numbers of the non-hub connectors responsible for the connectivity between the modules. Thus, the modules in these two groups are apparently worse separated from each other than, for example, in the $\mathrm{CMV}^{-}$males. The network of the $\mathrm{CMV}^{+} \mathrm{fe}-$ males (D) contains two hubs and eight non-hub connectors, and thus demonstrates a modular structure with moderate number of hubs and connectors. Note also that all cognitive nodes in this group are provincial nodes and therefore play a secondary role in the network. In summary, it can be stated that the networks under consideration exhibit a different balance between intra- and inter-modular information flow with different numbers of hub and connector nodes playing a significant role for this balance and for network functioning. Which of these types of modular organization is more effective, remains to be investigated.

\section{Discussion}

There is a growing body of evidence supporting the notion that the immune system is not hermetically selfregulated but functions in intimate interrelations with other physiological systems, including the nervous system $[5,28]$. These interactions are present at the various levels of organization - at the local, as well as at the whole organism level - by sharing a common language of a wide range of cytokines, receptor molecules, hormones, neuropeptides, metabolic and neurotrophic factors allowing cross-communication [29, 30]. Particularly in the process of aging, this reciprocal cross-talk may under certain circumstances permit augmentation of maladaptive inflammatory loops, which could disturb homeostasis and contribute to the age-related functional alterations or even to pathological conditions [2, 31-33].

Several analytical techniques to investigate these interactions have been established so far, but our understanding of the interplay between different factors in such interrelated processes is still in its infancy. Despite some progress, there is a further need to place the data from different physiological and functional levels in a biological context with the aim of interpreting their multifaceted orchestration as a whole. Many studies highlight the role of different inflammatory cytokines in the low-grade inflammation, dubbed "inflammaging", and the importance of pro-inflammatory and anti-inflammatory homeostasis for cognitive health in aging $[17,18,34-36]$. Additionally, the interrelated effects of inflammatory factors and their influence on neuroimmune and neuroendocrine functions can be modified by the chronic immune activity required to control lifelong persistent CMV infection [2, 37]. In the present work, we propose a strategy for quantitative description of multiple interactions between different cytokines, receptor molecules, metabolic and neurotrophic factors, hormones, immune cells, and measures of cognitive performance with the help of a graphtheoretical approach. To the best of our knowledge, simultaneous network analyses of multiple inflammation-related mediators and cognitive performance in older CMV-seropositive and CMVseronegative men and women have not been previously accomplished.

Aging is associated with modulatory effects on the immune system - resulting in the universal, multifactorial changes, known as immunosenescence. This leads to functional changes in the immune cells, which produce more inflammatory cytokines and less anti-inflammatory mediators. CMV-persistence is associated with constant chronic stimulation of the immune system that could further contribute to induction and accumulation of the specific immune cell phenotypes known to be generally associated with immunosenescence. The fact that CMV has considerable influence on immunosenescence was first described 20 years ago [38] and has continuously been supported by numerous studies since then $[15,16,39-44]$. In the large-scale immune profiling and functional analysis of normal aging, it was impressively shown that the immune system alterations (determined as a number of significantly affected analytes) caused specifically by CMV were comparable to the differences seen between the sexes [45]. A lifelong persistent infection influences immune aging and can significantly modify the course of cognitive aging by acting in combination with individual differences in cytokine release [37, 46-48]. The modulatory effect of CMV-latency and sex were also demonstrated in our previous study [22]. Therefore, for the network analyses in the present study, we separated the participants into four groups according to their CMV-serostatus and sex.

We found that the modulatory impact of CMV and sex was also reflected in the specific differences of the network structure and the network topology dynamics observed between the four groups. In particular, $\mathrm{CMV}^{-}$ males were characterized through several strong connections between nodes of the pro-inflammatory cytokines IL-1 $\beta$, TNF, IL-18 and cognitive nodes including variables of episodic memory and fluid intelligence. Currently available evidence shows that pro-inflammatory cytokines exert a dose-dependent physiological neuroprotective but can however also mediate pathological neurodegenerative effects under certain circumstances 
[18]. IL-1 $\beta$ and TNF were demonstrated to have such a dual function, acting on the one hand as proinflammatory factors and on the other as neuromodulators, subserving memory and other cognitive processes. In other words, they not only play a role in neuroinflammation, but (at their low concentrations) also in complex processes such as synaptic plasticity, neurogenesis, longterm potentiation and memory consolidation $[34,35]$.

Less strong but numerous connections were found between nodes of the anti-inflammatory cytokines and cognition in the network of $\mathrm{CMV}^{-}$males. This is partly in line with our previous findings on the positive association of episodic memory with the anti-inflammatory cytokine IL-10 in the $\mathrm{CMV}^{-}$elderly men and women [22]. IL-10 is known to have a neuroprotective role due to its inhibitory action on inflamed microglia [17]. The same $\mathrm{CMV}^{-}$male group also has significantly elevated levels of anti-inflammatory IL-10 and sTNF-R as well as reduced levels of pro-inflammatory cytokines in their peripheral circulation, as reported in our recent study [22]. Having this information in mind, we can speculate that strong connections between cognitive nodes and the nodes of (low-levels) pro-inflammatory cytokines on the one hand and numerous connections of cognition to the nodes of the (high-level) anti-inflammatory cytokines on the other, could possibly explain the cognitive advantage in the fluid intelligence and working memory found for this group of participants in our previous work [22]. Remarkably, this was the only group in which nodes of pro- and anti-inflammatory cytokines had no direct connections to each other. The other three groups, (two of which, $\mathrm{CMV}^{-}$females and $\mathrm{CMV}^{+}$males, were characterized in our previous study by heterogeneously unbalanced levels of pro- and anti-inflammatory mediators and by an adverse metabolic environment) demonstrated, in contrast, various more or less strong connections between pro- and anti-inflammatory cytokines, which were probably important and necessary homeostatic responses to these unbalanced peripheral conditions. In our previous study, the network of $\mathrm{CMV}^{+}$ women (that shows multiple connections between nodes of pro- and anti-inflammatory cytokines), exhibited significantly higher levels of the anti-inflammatory factors sTNF-R and IL-1RA. We also found previously that in the $\mathrm{CMV}^{+}$group, fluid intelligence, episodic and working memory were negatively associated with the antiinflammatory factor IL-1RA, the level of which was assumed to be simultaneously increased as a reaction to the elevation of the pro-inflammatory cytokines in the periphery [22]. This phenomenon has also been reported by other investigators $[33,49,50]$, showing that individuals with high levels of pro-inflammatory cytokines also tend to display elevated levels of anti-inflammatory factors. The network analyses in the present study allowed the visualization of these multiple and mutual connections between pro- and anti-inflammatory biomarkers, which were only assumed in our previous work [22].

Interestingly, the network of $\mathrm{CMV}^{-}$males demonstrated some direct connections between DHEA and cognitive nodes, and also to the nodes of antiinflammatory and metabolic factors. The $\mathrm{CMV}^{+}$males, in contrast, displayed multiple connections to cognitive nodes, but no connections to anti-inflammatory nodes, and were connected to the inflammatory cytokine IL-6. A completely different picture was seen in $\mathrm{CMV}^{-}$females with no connections of DHEA either to proinflammatory cytokines or cognition, whereas $\mathrm{CMV}^{+}$ women had multiple connections to nodes of cytokines and cognition. It is known that inflammatory reactions are, in general, under the influence of different mechanisms including neuroendocrine interactions. Proinflammatory mediators and cytokines may lead to the activation of the hypothalamic-pituitary-adrenal axis (HPA) that is in turn capable of modulating the process of inflammation [51-55]. DHEA and cortisol are multifunctional adrenocortical hormones with such immunomodulatory properties. They exert potent and broad influences throughout the body and brain and jointly impact on a variety of processes related to metabolic, immune, and cognitive functions [52]. Being especially abundant in the brain, DHEA exerts a protective effect against the deterioration of mental functioning with aging. Interestingly, both cortisol and DHEA in the $\mathrm{CMV}^{-}$males are non-hub connectors exhibiting numerous links to diverse modules in the modular organization of the network. This indicates that these nodes play a crucial role in communication between different subsystems. Inverse correlations between DHEA concentrations and neuroinflammatory-related diseases have repeatedly been found in the elderly [52, 56-58]. Similar to DHEA, the cortisol nodes in our study displayed very heterogeneous and group-specific picture concerning their connections. Whereas $\mathrm{CMV}^{-}$males showed connections from cortisol to the nodes of pro-inflammatory TNF, IGF-1, IGFBP-3, metabolic factors, and immune cells, the cortisol-node of $\mathrm{CMV}^{-}$females had only one connection to IL-18. In the $\mathrm{CMV}^{+}$groups, men showed weak but multiple cortisol-connections to cognitive nodes, neurotrophins, pro- and anti-inflammatory factors. In the network of women, cortisol was connected only to the metabolic factors. The heterogeneous picture seen in these connections may partly be due to the fact that although the effect of cortisol has been typically shown to be immunosuppressive, at certain concentrations it can also induce a biphasic response during a later, delayed systemic inflammatory response [59] through augmentation of inflammation [53]. In other 
words, the regulation of inflammation by cortisol may vary from anti- to pro-inflammatory in a time- and concentration-dependent manner and this contributes to further complexity in interpreting results of these already complex interactions.

Pro-inflammatory cytokines are known to be involved in dynamic interactions with the main neurotrophic factor, IGF-1 and its regulator, IGFBP-3 by decreasing IGF1 signaling and by enhancing the production of IGFBP3. Conversely, IGF-1 is capable of depressing proinflammatory cytokine signaling by increasing antiinflammatory IL-10 secretion and by directly depressing pro-inflammatory cytokine signaling $[23,60,61]$. Both IGF-1 and IGFBP-3 had relative strong connections to metabolic nodes in the $\mathrm{CMV}^{-}$men, but only one weak connection to CRP. In contrast, all three of the other networks displayed multiple connections to both proand anti-inflammatory cytokines - possibly due to their involvement in the dynamic interactions aiming to balance the pro- and anti-inflammatory equilibrium. Concerning the connections between neurotrophins and cognitive nodes, we can see a relative homogeneous picture: with some connections in the networks of CMVnegative and -positive men, and with only one connection in the networks of CMV-negative and -positive women. There is substantial evidence that IGF-1 deficiency represents a contributing factor for reduced cognitive abilities in aged humans [57, 62], and that supplementation with IGF-1 may reverse this deficit [60, 63-66]. Measures of circulating IGF-1, IGFBP-3 and their ratio, have been proposed for monitoring aged individuals and those at risk of cognitive and functional decline [62]. Thus, we can speculate that the relatively low number of connections between neurotrophins and cognitive nodes, seen in all four networks, might be due to the overall age-related decrease of these neurotrophic factors in peripheral circulation of elderly participants.

Our study has many strengths, including that it is one of the first studies to extensively characterize, prior to any physical, cognitive, and combine interventions, the network topology dynamics in multiple peripheral circulating biomarkers and markers of cognitive functioning. Applying a graph-theory approach allowed us not only to visualize biologically meaningful interconnections between nodes but also for the first time to compare the network topology metrics between different groups of CMV-seronegative and -positive men and women in a statistically sound manner. Inspection of separate nodes in the networks showed that these network topology differences were especially strong for cytokines and cognitive nodes. Modularity analyses showed that the networks under consideration exhibited highly differentiated modular organization in all cases. Moreover, we found that all four networks represented so-called small- world networks (SWNs) at all levels of wiring costs and were identified as SWNs with more random characteristics. We found that the network of the $\mathrm{CMV}^{-}$males contains more hub nodes but fewer connector nodes than the other three groups. This indicates that the modules in this participants' group are more autonomous and the information flow between the modules may be realized through a small number of connector nodes. Interestingly, three of the four hubs are cognitive variables and the fourth one is IGFBP-3. Thus, cognitive nodes, such as fluid intelligence, working memory, and perceptual speed play a central role in the network of $\mathrm{CMV}^{-}$males driving or controlling the connections within the corresponding modules.

This is the first study investigating the segregation and integration properties of the individual networks of CMV-seropositive and -negative older men and women by analyzing such network topology measures as clustering coefficient, characteristic path length, local and global efficiency. Using the rewiring procedure for network analyses, we compared network topology dynamics and found that mean clustering coefficient was highest and $C P L$ shortest in the network of the $\mathrm{CMV}^{-}$males. The same network also manifested the highest local and global efficiency, allowing it to be identified as the network with optimal features of segregation and integration. In our previous study, the same group of participants displayed the most balanced inflammatory status in their peripheral circulation (with low levels of proinflammatory cytokines and high levels of antiinflammatory biomarkers) as well as significantly higher cognitive performance in working memory and fluid intelligence [22]. Further studies, however, are required to confirm these findings and to better understand such complex relationships and network topology changes between different groups of older CMV-seropositive and -negative men and women.

There are several limitations to our study that should be acknowledged. The first one has already been mentioned in our previous publication and is "related to the fact that our pre-training cohort consisted of relatively healthy, non-obese, and well-educated Berlin residents with a comparatively low seroprevalence for CMV for this age. For this reason, the generalizability of some of our findings may be limited to the Berlin healthy aging population or to a similar European population in urban areas" [22]. The next limitation concerns the fact that we were not able to disentangle the potential effect of age on the circulating biomarkers and cognitive performance due to the fact that our pre-training cohort consisted exclusively of aged participants with a rather narrow age range from 64 to 79 years old. Another limitation is related to the exploratory character of our study of the network patterns and their relationships. We are 
well aware that our choice of variables in the present study, selected on the basis of their involvement in the known age-related functional alterations in the immune, nervous, and other central physiological systems, does not necessarily cover all potential players and, we therefore need further more extended network analyses to obtain a more comprehensive picture on their dynamic interactions.

\section{Conclusions}

Network analyses applying a graph-theoretical approach provide a useful strategy for visualization and quantitative description of multiple interactions between various circulating pro- and anti-inflammatory biomarkers, hormones, neurotrophic and metabolic factors, immune cells, and measures of cognitive performance and can be in general applied for analyzing interactions between different physiological systems. Applying this approach, we were able to confirm our previous findings that CMVinfection and sex modulate multiple circulating biomarkers and cognitive performance and that balanced inflammatory and metabolic status in elderly contributes to better cognitive performance. Analyzing the network topology dynamics of circulating biomarkers and cognitive performance in older CMV-seropositive and -seronegative men and women we were able to show that highly integrated and segregated networks have optimal neuroimmune and cognitive interactions.

\section{Methods}

\section{Participants}

The sample has already been described in [22]. It consisted of 161 older adults (Fig. 1) who had enrolled in a training study that included physical, cognitive, and combined training interventions. Male and female subjects were recruited from volunteer participant pools at the Max Planck Institute for Human Development and by advertisements in the metropolitan area of Berlin, Germany. All the volunteers lived independently at home, leading an active life. Participants were healthy, right-handed adults, aged 64-79 years. All volunteers completed a medical assessment prior to data collection. The medical examination was conducted at the Charité Sports Medicine, Charité Universitätsmedizin Berlin. Of the originally recruited 201 volunteers only 179 individuals met inclusion criteria for study participation after medical assessment. None of the participants had a history of head injuries, medical (e.g., heart attack), neurological (e.g., epilepsy), or psychiatric (e.g., depression) disorders. None of the volunteers had suffered from chronic inflammatory, autoimmune or cancer diseases, nor had clinically evident infections. Moderately elevated and controlled blood pressure was not considered as an exclusion criterion. All subjects completed the informed consent form to the study protocol, which was approved by the Ethics Committee of the German Society of Psychology, UL 072014.

\section{Circulating biomarkers assessment}

The assessment of circulating cytokines, receptor antagonist, soluble cytokine receptor, and CMV-serostatus has been described in detail [22]. The blood used for testing of peripheral biomarkers was collected during a medical examination in the timeframe between $11 \mathrm{am}$ and $2 \mathrm{pm}$. For all analyses, the participants were separated into four groups according to their CMVserostatus and sex (Fig. 1). The effective sample consisted of $29 \mathrm{CMV}$-negative males (mean age $=72.4, \mathrm{SD}=$ 3.5 , age range $=64.0-77.2), 30 \mathrm{CMV}$-negative females $($ mean age $=70.0, \mathrm{SD}=3.6$, age range $=64.1-76.9), 50$ CMV-positive males (mean age $=70.4, \mathrm{SD}=3.7$, age range $=64.0-78.1$ ), and $52 \mathrm{CMV}$-positive females (mean age $=70.2, \mathrm{SD}=3.6$, age range $=63.9-77.1$ )

\section{Cytokines TNF, IL-10, IL-6, and IL-1 $\beta$}

Serum levels of pro- and anti-inflammatory cytokines (TNF, IL-10, IL-6, and IL-1 $\beta$ ) were determined using the high-sensitivity cytometric bead array (CBA) flex system (BD Biosciences, San Jose, CA, USA) that allows multiplex quantification in a single sample. All analyses were performed according to the manufacturer's instructions; to increase accuracy, an additional standard dilution was added. The fluorescence produced by CBA beads was measured on a BD FACS CANTO II Flow Cytometer and analyzed using the software FCAP Array v3 (BD Biosciences).

sTNF-R, IL-1RA, IL-18, cortisol, and DHEA levels, and CMVserostatus

To gauge sTNF-R (80 kDA), IL-1RA, and IL-18 levels, we used the Sandwich Enzyme-linked Immunosorbent Assay (ELISA), a sensitive method allowing for the measurement of an antigen concentration in an unknown sample. All analyses were conducted according to the manufacturer's instructions. The levels of human circulating sTNF-R ( $80 \mathrm{kDA})$, IL-1RA, and IL-18 were determined using the Platinum ELISA kit for the quantitative detection of the three cytokines (ThermoFisher SCIENTIFIC Invitrogen, Vienna, Austria, catalog numbers: BMS211, BMS2080 and BMS267/2).

Serum levels of anti-Cytomegalovirus IgG were determined using a commercial ELISA kit (IBL International GMBH, Hamburg, Germany, catalogue number: RE57061) and according to the manufacturer's instructions. Samples were considered to give a positive signal if the absorbance value exceeded $10 \%$ over the cut-off, whereas a negative signal was absorbance lower than $10 \%$ below the cut-off. 
Quantitative determination of Cortisol and DHEA in serum of participants was performed using Human Cortisol and Human DHEA (sulfate form) ELISA kits (Qarigo Biolabatories, catalog number: ARG81162 and ARG80837). The central mechanism of the competitive ELISA is a competitive binding process performed by sample antigen and add-in antigen. The amount of bound add-in antigen is inversely proportional to the concentration of the sample antigen. The analyses were performed according to the manufacturer's instructions.

All samples were assessed in duplicate at 450 or 450 / $620 \mathrm{~nm}$ using a Multiscan-FC Microtiter Plate Photometer. Protein concentrations were determined in relation to a four-parameter standard curve (Prism 8 GraphPad, San Diego, CA, USA) or calculated using Microsoft Excel 2011.

\section{Levels of IGF-1 and IGFBP-3, CRP, metabolic factors, and immune cells}

Serum levels of Insulin-like growth factor 1 (IGF-1) and Insulin-Like Growth Factor-Binding Protein 3 (IGFBP-3) were determined at the Endocrine Routine Laboratory (University Hospital of Würzburg). Measurement of IGF-1 (L2KIGF2) and IGFBP-3 (L2KGB2) was performed according to the manufacturer's instruction, using the Immulite 2000 system - an automated solidphase, Electrochemiluminescence-Immunoassay (ECLIA) from Siemens Healthcare (Germany). Levels of Creactive protein (CRP), cholesterol, LDL, HDL, triglyceride, lymphocytes, leukocytes, monocytes, and neutrophils were measured within the clinical diagnostics facility of Berlin, Labor28. Serum concentrations of cholesterols and triglyceride were measured using enzymatic colorimetric tests (Roche, Basel, Switzerland). Counts of the immune cells were determined by flow cytometry (Sysmex, Norderstedt, Germany).

\section{Cognitive assessment}

Cognitive assessment was performed 3 months after blood collection, immediately before beginning of training. Participants were invited to a baseline session that lasted about $3.5 \mathrm{~h}$, in which they were tested in groups of four to six individuals. The cognitive battery included a broad range of measures of learning and memory performance, processing speed, working memory, and executive functioning. The group received a standardized session protocol and started, after instructions, each task with practice trials to ensure that all participants understood the task. Responses were collected via button boxes, the computer mouse, or the keyboard. A detailed description of the tasks and scores used in the present study is included in the supplementary material.

\section{Network construction and network properties}

For network construction, we used a coefficient of determination $\left(R^{2}\right)$, ranging between 0 and 1 , and indicating the extent to which one dependent variable is explained by the other. The coefficient of determination was calculated between all pairs of variables $(N=33)$ for the four experimental groups separately. Thus, the common network in each of the groups contained 33 nodes altogether, covering all possible interactions between the variables or nodes. To be able to construct sparse networks with relatively stable network topology, we first investigated ordered (lattice) and random networks containing the same number of nodes and edges as the real network. To do so, we randomized the edges in the real network to achieve a random network. As for the lattice network, we redistributed the edges such that they were laying close to the main diagonal and in the corner opposite to the main diagonal with increasing order of their weights. The lattice network reconstructed in such a way has the same number of nodes and edges as the initial real network but is characterized by ring or lattice topology incorporating nearest-neighbor connectivity [67]. Random networks were constructed 100 times, and the network topology measures determined each time were averaged for further analyses. To investigate the network topology of the real networks in topology space between regular and random networks with different wiring cost levels, we constructed real and control (i.e., lattice and random) networks in the range of costs between 10 and $60 \%$ with a step of $1 \%$ of wiring costs (the ratio of the number of actual connections to the maximum possible number of connections in the network). We then decided to set the cost level to $25 \%$, which resulted in sparse and at the same time stable network topology.

\section{Degrees and strengths}

The degree of a node provides information about the number of links connected to that node, and the strength reflects the overall strength of that node's connections or weights. Thus, the strength could be considered as a weighted degree. Degree or strength of a node indicates the activity of that node, whereas the sum or mean of all degrees (strengths) represents the overall activity of the network. As $R^{2}$ is a weighted symmetric measure, we obtained the node's strength $\left(S_{i}^{w}\right)$ as the sum of weights of all connections $\left(w_{i j}\right)$ to node $i$, and calculated the mean strength $(S)$ across all nodes in the network:

$$
S=\frac{1}{N} \sum_{i \in N} S_{i}^{w}=\frac{1}{N} \sum_{i, j \in N} w_{i j}
$$

\section{Clustering coefficient and characteristic path length}

For an individual node $i$, the clustering coefficient $\left(C C_{i}^{w}\right)$ is defined as the proportion of the number of existing 
neighbor-neighbor connections to the total number of possible connections within its neighborhood. In the case of a weighted graph, the mean $C C$ is calculated as follows [68]:

$$
C C=\frac{1}{N} \sum_{i \in N} C C_{i}^{w}=\frac{1}{N} \sum_{i \in N} \frac{2 t_{i}^{w}}{k_{i}\left(k_{i}-1\right)}
$$

with $t_{i}^{w}=\left(w_{i j} w_{i h} w_{j h}\right)^{1 / 3}$ being the number of weighted closed triangles around a node $i$; $k_{i}$ is the degree of the node $i$, and $N$ is the number of nodes in the network, $N=33$. The $C C$ measures the cliquishness of a typical neighborhood and is thus a measure of network segregation.

The shortest path length or distance $d_{i j}$ between two nodes $i$ and $j$ is normally defined as the minimal number of edges that have to be passed to go from $i$ to $j$. As our networks are weighted graphs, the weight of the links must be considered. The input matrix is then a mapping from weight to length (i.e., a weight inversion), and the distance $d_{i j}^{w}$ is the minimal weighted distance between the nodes $i$ and $j$, but not necessarily the minimal number of edges. To calculate the characteristic path length $(C P L)$ of a network, path lengths between all possible pairs of vertices or nodes in the network were determined [69] and then averaged among nodes:

$$
C P L=\frac{1}{N} \sum_{i \in N} L^{w}{ }_{i}=\frac{1}{N} \sum_{i \in N} \frac{\sum_{j \in N, j \neq i} d_{i j}^{w}}{N-1}
$$

whereby $L_{i}{ }^{w}$ is the shortest path length of a node $i$, and $N$ is the total number of nodes in the network. CPL shows the degree of network integration, with a short $C P L$ indicating higher network integration.

\section{Local and global efficiency}

Local efficiency ( $\left.E_{\text {local }}\right)$ is similar to the $C C$ and is calculated as the harmonic mean of neighbor-neighbor distances [70]:

$$
\begin{aligned}
E_{\text {local }} & =\frac{1}{N_{G_{i}}\left(N_{G_{i}}-1\right)} \sum_{i \in N} E_{\text {local }(i)}^{w} \\
& =\frac{1}{N_{G_{i}}\left(N_{G_{i}}-1\right)} \sum_{i \in N} \frac{1}{L_{j, h}}
\end{aligned}
$$

where $N_{G_{i}}$ is the number of nodes in subgraph $G_{i}$, comprising all nodes that are immediate neighbours of the node $i$ (excluding the node $i$ itself), and $E_{\text {local }(i)}^{w}$ is local efficiency of the node $i$ determined as the reciprocal of the shortest path length between neighbours $j$ and $h$. Thus, $E_{\text {local }}$ of node $i$ is defined with respect to the subgraph comprising all of $i$ 's neighbours, after removal of node $i$ and its incident edges (Latora and Marchiori, 2001). Like $C C, E_{\text {local }}$ is a measure of the segregation of a network, indicating efficiency of information transfer in the immediate neighbourhood of each node.

Global efficiency $\left(E_{\text {global }}\right)$ is defined as the average inverse shortest path length and is calculated by the formula [70]:

$$
E_{\text {global }}=\frac{1}{N} \sum_{i \in N} E_{\text {global }(i)}^{w}=\frac{1}{N} \sum_{i \in N} \frac{\sum_{j \in N, j \neq i}\left(d_{i j}^{w}\right)^{-1}}{N-1}
$$

whereby $E_{\text {global }(i)}^{w}$ is a nodal efficiency, $d_{i j}^{w}$ is the minimal weighted distance between the nodes $i$ and $j$, and $N$ is the total number of nodes in the network. The nodal efficiency is practically the normalized sum of the reciprocal of the shortest path lengths or distances from a given node to all other nodes in the network. Nodal efficiency quantifies how well a given node is integrated within the network, and global efficiency indicates how integrated is the common network. Thus, like CPL, $E_{\text {global }}$ is a measure of the integration of a network, but whereas $C P L$ is primarily influenced by long paths, $E_{\text {global }}$ is primarily influenced by short ones.

\section{Small-Worldness (SW) coefficients}

Using graph metrics determined for real and control (i.e., regular and random) networks, specific quantitative small-world metrics were obtained. The first smallworld metric, the so-called small-world coefficient $\sigma$, is related to the main metrics of a random graph $\left(C C_{\text {rand }}\right.$ and $C P L_{\text {rand }}$ ) and is determined on the basis of two ratios $\gamma=C C_{\text {real }} / C C_{\text {rand }}$ and $\lambda=C P L_{\text {real }} / C P L_{\text {rand }}$ [71]:

$$
\sigma=\frac{\gamma}{\lambda}=\frac{C C_{\text {real }} / C C_{\text {rand }}}{C P L_{\text {real }} / C P L_{\text {rand }}}
$$

The small-world coefficient $\sigma$ should be greater than 1 in the small-world networks (SWNs). The second SW metric, the so-called small-world coefficient $\omega$, is defined by comparing the characteristic path length of the observed (real) and random networks, and comparing the clustering coefficient of the observed or real network to that of an equivalent lattice (regular) network [72]:

$$
\omega=\frac{C P L_{\text {rand }}}{C P L_{\text {real }}}-\frac{C C_{\text {real }}}{C C_{\text {latt }}}
$$

This metric ranges between -1 and +1 and is close to zero for $\mathrm{SWN}\left(C P L_{\text {real }} \approx C P L_{\text {rand }}\right.$ and $\left.C C_{\text {real }} \approx C C_{\text {latt }}\right)$. Thereby, negative values indicate a graph with more regular properties $\left(C P L_{\text {real }}>>C P L_{\text {rand }}\right.$ and $C C_{\text {real }} \approx$ $C C_{\text {latt }}$, and positive values of $\omega$ indicate a graph with more random properties $\left(C P L_{\text {real }} \approx C P L_{\text {rand }}\right.$ and $C C_{\text {real }}<$ $\left.<C C_{\text {latt }}\right)$. As suggested in [72], the metric $\omega$ compared to $\sigma$ has a clear advantage, i.e., the possibility to define how much the network of interest resembles its regular or random equivalents. 


\section{Modularity analyses and Z-P parameter space}

To investigate the modular organization of the network and the individual role of each node in the emerging modularity or community structure, we partitioned the networks into modules applying modularity optimization algorithm and determined indices of modularity $(Q)$, within-module degree $\left(Z_{i}\right)$, and participation coefficient $\left(P_{i}\right)$ using the Brain Connectivity Toolbox [73]. The optimal community structure is a subdivision of the network into non-overlapping groups of nodes in a way that maximizes the number of within-module edges, and minimizes the number of between-module edges. $Q$ is a statistic that quantifies the degree to which the network may be subdivided into such clearly delineated groups or modules. It is given for weighted networks by the formula [74]:

$$
Q^{w}=\frac{1}{l^{w}} \sum_{j \in N}\left[w_{i j}-\frac{k_{i}^{w} k_{j}^{w}}{l^{w}}\right] \cdot \delta_{m_{i} m_{j}}
$$

where $l^{w}$ is the total number of edges in the network, $\mathrm{N}$ is the total number of nodes in the network, $w_{i j}$ are connection weights, $k_{i}^{w}$ and $k_{j}^{w}$ are weighted degrees or strengths of the nodes, and $\delta_{m_{i}, m_{j}}$ is the Kronecker delta, where $\delta_{m_{i}, m_{j}}=1$ if $m_{i}=m_{j}$, and 0 otherwise. High modularity values indicate strong separation of the nodes into modules. $Q^{w}$ is zero if nodes are placed at random into modules or if all nodes are in the same cluster. To test the modularity of the empirically observed networks, we compared them to the modularity distribution $(N=100)$ of random networks as described above [75].

The within-module degree $Z_{i}$ indicates how well node $i$ is connected to other nodes within the module $m_{i}$. As shown in Guimerà and Amaral [27], it is determined by:

$$
Z_{i}=\frac{k_{i}\left(m_{i}\right)-\bar{k}\left(m_{i}\right)}{\sigma^{k\left(m_{i}\right)}}
$$

where $k_{i}\left(m_{i}\right)$ is the within-module degree of node $i$ (the number of links between $i$ and all other nodes in $m_{i}$ ), and $\bar{k}\left(m_{i}\right)$ and $\sigma^{k\left(m_{i}\right)}$ are the mean and standard deviation of the within-module degree distribution of $m_{i}$.

The participation coefficient $P_{i}$ describes how well the nodal connections are distributed across different modules [27]:

$$
P_{i}=1-\sum_{m \in M}\left(\frac{k_{i}\left(m_{i}\right)}{k_{i}}\right)^{2}
$$

where $M$ is the set of modules, $k_{i}\left(m_{i}\right)$ is the number of links between node $i$ and all other nodes in module $m_{i}$, and $k_{i}$ is the total degree of node $i$ in the network. Correspondingly, $P_{i}$ of a node $i$ is close to 1 if its links are uniformly distributed among all the modules, and is zero if all of its links lie within its own module. $Z_{i}$ and $P_{i}$ values form a so-called $Z-P$ parameter space and are characteristic for the different roles of the nodes in the network [27]. These roles in the Z-P parameter space could be defined as follows: ultra-peripheral nodes $\left(P_{i}<\right.$ 0.05), provincial nodes (low $Z_{i}$ and $P_{i}$ values), connector nodes (low $Z_{i}$ and high $P_{i}$ values), hub nodes (high $Z_{i}$ and low $P_{i}$ values), and hub connector nodes (high $Z_{i}$ and $P_{i}$ values). In this context, hubs are responsible for intra-modular connectivity and contain multiple connections within a module, while connector nodes maintain inter-modular connectivity and are responsible for links between the modules.

\section{Statistical analysis}

In order to statistically compare the four different networks at a given cost level, we used a rewiring procedure with a step-by-step replacement of a non-existing edge through an existing one and consecutive determination network topology metrics each time. This procedure can specify the network stability and network topology alteration by very small changes in the network configuration. In a statistical sense, this procedure is similar to bootstrapping with replacement applied to time series. In total, there were about 50,000 rewired networks, on which mean and standard deviation (SD) of the network topology metrics were determined. Because the rewiring distribution showed a normal shape and a small bias, we were able to achieve a $99.7 \%$ confidence interval $(C I)$ for the mean by using the empirical rule: $C I=$ mean $\pm 3 \times$ $S D(P<0.005)$.

\section{Supplementary information}

Supplementary information accompanies this paper at https://doi.org/10. 1186/s12979-019-0171-x.

Additional file 1: Figure S1. (A) CC is greatest in lattice networks (blue) and lowest in random networks (green), whereas CC for the real networks (red) is in-between. In contrast, (B) CPL is shortest in random and longest in lattice networks, while the real networks are in-between. CMV, Cytomegalovirus; CMV m, CMV-seronegative men; $\mathrm{CMV}^{+} \mathrm{m}, \mathrm{CMV}$ seropositive men; CMV f, CMV-seronegative women; $\mathrm{CMV}^{+}$f, CMVseropositive women. Figure S2. (A) Local efficiency was highest in regular networks (at least for the cost levels under 45\%) and lowest in random networks (at least for the cost levels under 20\%), while (B) global efficiency was highest in random (green) and lowest in lattice (blue) networks practically for all levels of wiring costs, with real (red) networks were always in-between. CMV, Cytomegalovirus; CMV m, CMVseronegative men; $\mathrm{CMV}^{+} \mathrm{m}$, CMV-seropositive men; CMV f, CMVseronegative women; $\mathrm{CMV}^{+} \mathrm{f}, \mathrm{CMV}$-seropositive women.

\section{Abbreviations}

CBA: Cytometric bead array; CC: Clustering coefficient; Cl: Confidence interval; CMV: Cytomegalovirus; CPL: Characteristic path length; CRP: Creactive protein; DHEA: Dehydroepiandrosterone; $E_{\text {global: }}$ Global efficiency; ELISA: Enzyme-linked Immunosorbent Assay; Elocal. Local efficiency; EM: Episodic memory; Gf: Fluid intelligence; HDL: High-density lipoprotein; IGF-1: Insulin-like growth factor-1; IGFBP-3: IGF-binding protein; IgG: Immunoglobulin G; IL: Interleukin; IL-1RA: Interleukin 1 receptor antagonist; LDL: Low-density lipoprotein; sTNF-R: Soluble Tumor Necrosis Factor receptor; TNF: Tumor Necrosis Factor; WM: Working memory 


\section{Acknowledgments}

We would like to express our very great appreciation to Elisabeth Wenger for her valuable, constructive, and helpful suggestions during the study. We thank Sandra Düzel for providing cognitive data, reading manuscript, and her constructive remarks. We thank Marcel Gaetjen for his excellent methodological support in applying of the CBA-flex system and for providing the FCAP-Array-v3 software. We are thankful to the students of the Structural Plasticity Group for their great contribution in collecting the data reported above. We would like to thank Nadine Taube, Kirsten Becker, and Anke Schepers-Klingebiel for managing all organizational issues. We thank Carola Misgeld for medical data assessment and blood collection. We are grateful to all participants of the study.

\section{Authors' contributions}

Conceptualization: SDB, LM, GP, MS, and VM; methodology: VM, SDB; SR, and TD; software: VM; validation: VM, SR, SDB; TD, formal analysis: SDB, and VM; investigation: SDB, TD, and SR; writing-original draft preparation: SDB; writing-review and editing: GP, LM, VM, and SDB. All authors read and approved the final version of manuscript.

\section{Funding}

This research was supported by the Max Planck Society and is part of the BMBF-funded Energl consortium (01GQ1421B).

\section{Availability of data and materials}

The datasets for this study will not be made publicly available due to restrictions included in the consent statement that the participants of the study signed only allow the present data to be used for the research purposes within the Max Planck Institute for Human Development in Berlin.

\section{Ethics approval and consent to participate}

All participants completed the informed consent form to the study protocol which was approved by the Ethics Committee of the German Society of Psychology, UL 072014.

\section{Consent for publication}

The consent forms of all participants are held by the authors' institute. The data in this work have not been published elsewhere. All authors agree to submit this manuscript for publication in this journal.

\section{Competing interests}

The authors declare that the research was conducted in the absence of any commercial or financial relationships that could be construed as a potential conflict of interest.

\section{Author details}

${ }^{1}$ Max Planck Institute for Human Development, Berlin, Germany. ${ }^{2}$ University of Tübingen, Tübingen, Germany. ${ }^{3}$ Institute of Clinical Neurobiology, Würzburg, Germany. ${ }^{4}$ Department of Internal Medicine I, Division of Endocrinology and Diabetes, University Hospital of Würzburg, Würzburg, Germany.

Received: 4 July 2019 Accepted: 26 November 2019

Published online: 04 December 2019

\section{References}

1. Franceschi C, Garagnani P, Vitale G, Capri M, Salvioli S. Inflammaging and 'Garb-aging'. Trends Endocrinol Metab. 2017;28(3):199-212.

2. Di Benedetto S, Müller L, Wenger E, Duzel S, Pawelec G. Contribution of neuroinflammation and immunity to brain aging and the mitigating effects of physical and cognitive interventions. Neurosci Biobehav Rev. 2017;75: $114-28$.

3. Beydoun MA, Dore GA, Canas JA, Liang H, Beydoun HA, Evans MK, et al. Systemic inflammation is associated with longitudinal changes in cognitive performance among urban adults. Front Aging Neurosci. 2018;10:313.

4. Procaccini C, Pucino V, De Rosa V, Marone G, Matarese G. Neuro-endocrine networks controlling immune system in health and disease. Front Immunol. 2014;5:143.

5. Dantzer R. Neuroimmune interactions: from the brain to the immune system and vice versa. Physiol Rev. 2018;98(1):477-504.
6. Gottesman RF, Albert MS, Alonso A, Coker LH, Coresh J, Davis SM, et al. Associations between midlife vascular risk factors and 25-year incident dementia in the atherosclerosis risk in communities (ARIC) cohort. JAMA Neurol. 2017;74(10):1246-54.

7. Alboni S, Maggi L. Editorial: cytokines as players of neuronal plasticity and sensitivity to environment in healthy and pathological brain. Front Cell Neurosci. 2015;9:508

8. Ventura MT, Casciaro M, Gangemi S, Buquicchio R. Immunosenescence in aging: between immune cells depletion and cytokines up-regulation. Clin Mol Allergy. 2017;15:21.

9. Fülop $T$, Larbi A, Dupuis $G$, Le Page A, Frost EH, Cohen AA, et al. Immunosenescence and Inflamm-aging as two sides of the same coin: friends or foes? Front Immunol. 2017:8:1960.

10. De la Fuente M, Gimenez-Llort L. Models of aging of neuroimmunomodulation: strategies for its improvement. Neuroimmunomodulation. 2010;17(3):213-6.

11. Walker KA, Gottesman RF, Wu A, Knopman DS, Gross AL, Mosley TH Jr, et al. Systemic inflammation during midlife and cognitive change over 20 years: the ARIC study. Neurology. 2019;92(11):e1256-e67.

12. Du Y, Zhang G, Liu Z. Human cytomegalovirus infection and coronary heart disease: a systematic review. Virol J. 2018;15(1):31.

13. Garcia Verdecia B, Saavedra Hernandez D, Lorenzo-Luaces P, de Jesus Badia Alvarez T, Leonard Rupale I, Mazorra Herrera Z, et al. Immunosenescence and gender: a study in healthy Cubans. Immun Ageing. 2013;10(1):16.

14. Kilgour AH, Firth C, Harrison R, Moss P, Bastin ME, Wardlaw JM, et al. Seropositivity for CMV and IL-6 levels are associated with grip strength and muscle size in the elderly. Immun Ageing. 2013;10(1):33.

15. Pawelec G, Derhovanessian E. Role of CMV in immune senescence. Virus Res. 2011;157(2):175-9.

16. Pawelec G, McElhaney JE, Aiello AE, Derhovanessian E. The impact of CMV infection on survival in older humans. Curr Opin Immunol. 2012;24(4):50711.

17. Lobo-Silva D, Carriche GM, Castro AG, Roque S, Saraiva M. Balancing the immune response in the brain: IL-10 and its regulation. J Neuroinflammation. 2016;13(1):297.

18. Perry RT, Collins JS, Wiener $H$, Acton R, Go RC. The role of TNF and its receptors in Alzheimer's disease. Neurobiol Aging. 2001;22(6):873-83.

19. Nakagomi A, Seino Y, Noma S, Kohashi K, Kosugi M, Kato K, et al. Relationships between the serum cholesterol levels, production of monocyte proinflammatory cytokines and long-term prognosis in patients with chronic heart failure. Intern Med. 2014;53(21):2415-24.

20. Lee BK, Glass TA, McAtee MJ, Wand GS, Bandeen-Roche K, Bolla Kl, et al. Associations of salivary cortisol with cognitive function in the Baltimore memory study. Arch Gen Psychiatry. 2007;64(7):810-8.

21. Wersching H, Duning T, Lohmann H, Mohammadi S, Stehling C, Fobker M, et al. Serum C-reactive protein is linked to cerebral microstructural integrity and cognitive function. Neurology. 2010;74(13):1022-9.

22. Di Benedetto S, Gaetjen M, Muller L. The Modulatory Effect of Gender and Cytomegalovirus-Seropositivity on Circulating Inflammatory Factors and Cognitive Performance in Elderly Individuals. Int J Mol Sci. 2019;20(4).

23. O'Connor JC, McCusker RH, Strle K, Johnson RW, Dantzer R, Kelley KW. Regulation of IGF-I function by proinflammatory cytokines: at the interface of immunology and endocrinology. Cell Immunol. 2008;252(1-2):91-110.

24. Bhavnani SK, Victor S, Calhoun WJ, Busse WW, Bleecker E, Castro M, et al. How cytokines co-occur across asthma patients: from bipartite network analysis to a molecular-based classification. J Biomed Inform. 2011;44(Suppl 1):S24-30.

25. Müller $V$, Perdikis $D$, von Oertzen $T$, Sleimen-Malkoun $R$, Jirsa $V$, Lindenberger U. Structure and topology dynamics of hyper-frequency networks during rest and auditory oddball performance. Front Comput Neurosci. 2016;10:108.

26. Müller $V$, Jirsa $V$, Perdikis $D$, Sleimen-Malkoun $R$, von Oertzen $T$, Lindenberger $U$. Lifespan changes in network structure and network topology dynamics during rest and auditory oddball performance. Front Aging Neurosci. 2019;11:138.

27. Guimera R, Nunes Amaral LA. Functional cartography of complex metabolic networks. Nature. 2005;433(7028):895-900.

28. Müller L, Fulop T, Pawelec G. Immunosenescence in vertebrates and invertebrates. Immun Ageing. 2013;10(1):12.

29. Talbot S, Foster SL, Woolf CJ. Neuroimmunity. Annu Rev Immunol. 2016:34: 421-47. 
30. Morel PA, Lee REC, Faeder JR. Demystifying the cytokine network: mathematical models point the way. Cytokine. 2017;98:115-23.

31. Müller L, Pawelec $G$. Aging and immunity - impact of behavioral intervention. Brain Behav Immun. 2014;39:8-22.

32. Müller L, Pawelec $G$. As we age: Does slippage of quality control in the immune system lead to collateral damage? Ageing Res Rev. 2015;23(Pt A):116-23.

33. Kirk GD, Dandorf S, Li H, Chen Y, Mehta SH, Piggott DA, et al. Differential relationships among circulating inflammatory and immune activation biomediators and impact of aging and human immunodeficiency virus infection in a cohort of injection drug users. Front Immunol. 2017;8:1343.

34. McAfoose J, Baune BT. Evidence for a cytokine model of cognitive function. Neurosci Biobehav Rev. 2009;33(3):355-66.

35. Vitkovic L, Bockaert J, Jacque C. "Inflammatory" cytokines: neuromodulators in normal brain? J Neurochem. 2000;74(2):457-71.

36. Tangestani Fard M, Stough C. A review and hypothesized model of the mechanisms that underpin the relationship between inflammation and cognition in the elderly. Front Aging Neurosci. 2019;11:56.

37. Bennett JM, Glaser R, Malarkey WB, Beversdorf DQ, Peng J, Kiecolt-Glaser JK. Inflammation and reactivation of latent herpesviruses in older adults. Brain Behav Immun. 2012;26(5):739-46.

38. Looney RJ, Falsey A, Campbell D, Torres A, Kolassa J, Brower C, et al. Role of cytomegalovirus in the T cell changes seen in elderly individuals. Clin Immunol. 1999;90(2):213-9.

39. Derhovanessian E, Larbi A, Pawelec G. Biomarkers of human immunosenescence: impact of Cytomegalovirus infection. Curr Opin Immunol. 2009;21(4):440-5.

40. Di Benedetto S, Derhovanessian E, Steinhagen-Thiessen E, Goldeck D, Muller L, Pawelec G. Impact of age, sex and CMV-infection on peripheral T cell phenotypes: results from the Berlin BASE-II study. Biogerontology. 2015; 16(5):631-43.

41. Fulop T, Larbi A, Pawelec G. Human T cell aging and the impact of persistent viral infections. Front Immunol. 2013;4:271.

42. Haeseker MB, Pijpers E, Dukers-Muijrers NH, Nelemans P, Hoebe CJ, Bruggeman CA, et al. Association of cytomegalovirus and other pathogens with frailty and diabetes mellitus, but not with cardiovascular disease and mortality in psycho-geriatric patients; a prospective cohort study. Immun Ageing. 2013;10(1):30.

43. McElhaney JE, Zhou X, Talbot HK, Soethout E, Bleackley RC, Granville DJ, et al. The unmet need in the elderly: how immunosenescence, CMV infection, co-morbidities and frailty are a challenge for the development of more effective influenza vaccines. Vaccine. 2012;30(12):2060-7.

44. Solana R, Tarazona R, Aiello AE, Akbar AN, Appay V, Beswick M, et al. CMV and Immunosenescence: from basics to clinics. Immun Ageing. 2012;9(1):23.

45. Whiting CC, Siebert J, Newman AM, Du HW, Alizadeh AA, Goronzy J, et al. Large-scale and comprehensive immune profiling and functional analysis of Normal human aging. PLoS One. 2015;10(7):e0133627.

46. Nikolich-Zugich J, Goodrum F, Knox K, Smithey MJ. Known unknowns: how might the persistent herpesvirome shape immunity and aging? Curr Opin Immunol. 2017:48:23-30.

47. Weltevrede M, Eilers R, de Melker HE, van Baarle D. Cytomegalovirus persistence and T-cell immunosenescence in people aged fifty and older: a systematic review. Exp Gerontol. 2016;77:87-95.

48. Villacres MC, Longmate J, Auge C, Diamond DJ. Predominant type 1 CMVspecific memory T-helper response in humans: evidence for gender differences in cytokine secretion. Hum Immunol. 2004;65(5):476-85.

49. Morrisette-Thomas V, Cohen AA, Fulop T, Riesco E, Legault V, Li Q, et al. Inflamm-aging does not simply reflect increases in pro-inflammatory markers. Mech Ageing Dev. 2014;139:49-57.

50. Tegeler C, O'Sullivan JL, Bucholtz N, Goldeck D, Pawelec G, SteinhagenThiessen $E$, et al. The inflammatory markers CRP, IL-6, and IL-10 are associated with cognitive function--data from the Berlin aging study II. Neurobiol Aging. 2016;38:112-7.

51. Wolkow A, Aisbett B, Reynolds J, Ferguson SA, Main LC. Relationships between inflammatory cytokine and cortisol responses in firefighters exposed to simulated wildfire suppression work and sleep restriction. Physiol Rep. 2015;3(11).

52. Kamin HS, Kertes DA. Cortisol and DHEA in development and psychopathology. Horm Behav. 2017;89:69-85.

53. Marques AH, Silverman MN, Sternberg EM. Glucocorticoid dysregulations and their clinical correlates. From receptors to therapeutics. Ann N Y Acad Sci. 2009;1179:1-18.
54. Alves VB, Basso PJ, Nardini V, Silva A, Chica JE, Cardoso CR. Dehydroepiandrosterone (DHEA) restrains intestinal inflammation by rendering leukocytes hyporesponsive and balancing colitogenic inflammatory responses. Immunobiology. 2016;221(9):934-43.

55. Wu Z, Li L, Zheng LT, Xu Z, Guo L, Zhen X. Allosteric modulation of sigma-1 receptors by SKF83959 inhibits microglia-mediated inflammation. J Neurochem. 2015;134(5):904-14.

56. Shields GS, Moons WG, Slavich GM. Inflammation, self-regulation, and health: an immunologic model of self-regulatory failure. Perspect Psychol Sci. 2017;12(4):588-612.

57. Willis EL, Wolf RF, White GL, McFarlane D. Age- and gender-associated changes in the concentrations of serum TGF-1 beta, DHEA-S and IGF-1 in healthy captive baboons (Papio hamadryas anubis). Gen Comp Endocrinol. 2014;195:21-7.

58. Wilson CJ, Finch CE, Cohen HJ. Cytokines and cognition--the case for a head-to-toe inflammatory paradigm. J Am Geriatr Soc. 2002;50(12):2041-56.

59. Elenkov IJ. Neurohormonal-cytokine interactions: implications for inflammation, common human diseases and well-being. Neurochem Int. 2008;52(1-2):40-51.

60. Ashpole NM, Sanders JE, Hodges EL, Yan H, Sonntag WE. Growth hormone, insulin-like growth factor-1 and the aging brain. Exp Gerontol. 2015;68:7681.

61. Junnila RK, List EO, Berryman DE, Murrey JW, Kopchick JJ. The GH/IGF-1 axis in ageing and longevity. Nat Rev Endocrinol. 2013:9(6):366-76.

62. Wennberg AMV, Hagen CE, Machulda MM, Hollman JH, Roberts RO, Knopman DS, et al. The association between peripheral total IGF-1, IGFBP-3, and IGF-1/IGFBP-3 and functional and cognitive outcomes in the Mayo Clinic study of aging. Neurobiol Aging. 2018;66:68-74.

63. Deijen JB, Arwert LI, Drent ML. The GH/IGF-I Axis and cognitive changes across a 4-year period in healthy adults. ISRN Endocrinol. 2011;2011:249421.

64. Arwert LI, Veltman DJ, Deijen JB, van Dam PS, Drent ML. Effects of growth hormone substitution therapy on cognitive functioning in growth hormone deficient patients: a functional MRI study. Neuroendocrinology. 2006;83(1): 12-9.

65. Molina DP, Ariwodola OJ, Weiner JL, Brunso-Bechtold JK, Adams MM. Growth hormone and insulin-like growth factor-I alter hippocampal excitatory synaptic transmission in young and old rats. Age (Dordr). 2013; 35(5):1575-87.

66. Bozdagi O, Tavassoli T, Buxbaum JD. Insulin-like growth factor-1 rescues synaptic and motor deficits in a mouse model of autism and developmental delay. Mol Autism. 2013;4(1):9.

67. Sporns $\mathrm{O}$, Honey CJ, Kotter R. Identification and classification of hubs in brain networks. PLoS One. 2007;2(10):e1049.

68. Fagiolo G. Clustering in complex directed networks. Phys Rev E Stat Nonlinear Soft Matter Phys. 2007;76(2 Pt 2):026107.

69. Watts DJ, Strogatz SH. Collective dynamics of 'small-world' networks. Nature. 1998;393(6684):440-2.

70. Latora V, Marchiori M. Efficient behavior of small-world networks. Phys Rev Lett. 2001;87(19):198701.

71. Humphries MD, Gurney K, Prescott TJ. The brainstem reticular formation is a small-world, not scale-free, network. Proc Biol Sci. 2006;273(1585):503-11.

72. Telesford QK, Joyce KE, Hayasaka S, Burdette JH, Laurienti PJ. The ubiquity of small-world networks. Brain Connect. 2011;1(5):367-75.

73. Rubinov M, Sporns O. Complex network measures of brain connectivity: uses and interpretations. Neuroimage. 2010;52(3):1059-69.

74. Newman ME. Analysis of weighted networks. Phys Rev E Stat Nonlinear Soft Matter Phys. 2004;70(5 Pt 2):056131.

75. Bassett DS, Khambhati AN. A network engineering perspective on probing and perturbing cognition with neurofeedback. Ann N Y Acad Sci. 2017; 1396(1):126-43.

\section{Publisher's Note}

Springer Nature remains neutral with regard to jurisdictional claims in published maps and institutional affiliations. 